Article

\title{
The Association between Green and Black Tea Consumption on Successful Aging: A Combined Analysis of the ATTICA and MEDiterranean ISlands (MEDIS) Epidemiological Studies
}

\author{
Nenad Naumovski ${ }^{1,2}$ (D), Alexandra Foscolou ${ }^{3}$, Nathan M. D'Cunha ${ }^{1,2}$, Stefanos Tyrovolas ${ }^{3,4}$, \\ Christina Chrysohoou ${ }^{5}$, Labros S. Sidossis ${ }^{3,6}$, Loukianos Rallidis ${ }^{7}$, Antonia-Leda Matalas ${ }^{3}$, \\ Evangelos Polychronopoulos ${ }^{3}$, Christos Pitsavos ${ }^{5}$ and Demosthenes Panagiotakos ${ }^{1,2,3,6, * \text { (D) }}$ \\ 1 Faculty of Health, University of Canberra, 2617 Canberra, Australia; \\ Nenad.Naumovski@canberra.edu.au (N.N.); Nathan.D'Cunha@canberra.edu.au (N.M.D.) \\ 2 Collaborative Research in Bioactives and Biomarkers (CRIBB) Group, University of Canberra, 2617 Bruce, \\ Australia \\ 3 Department of Nutrition and Dietetics, School of Health Science and Education, Harokopio University, \\ 17676 Athens, Greece; alexandra.foscolou@gmail.com (A.F.); s.tyrovolas@pssjd.org (S.T.); \\ lss133@rci.rutgers.edu (L.S.S.); amatala@hua.gr (A.-L.M.); evpol@hua.gr (E.P.) \\ 4 Parc Sanitari Sant Joan de Déu, Fundació Sant Joan de Déu, CIBERSAM, Universitat de Barcelona, \\ 08007 Barcelona, Spain \\ 5 First Cardiology Clinic, School of Medicine, University of Athens, 10679 Athens, Greece; \\ chrysohoou@usa.net (C.C.); cpitsavo@med.uoa.gr (C.P.) \\ 6 Department of Kinesiology and Health, School of Arts and Sciences, Rutgers University, NJ 08901, USA \\ 7 Second Cardiology Clinic, School of Medicine, University of Athens, 10679 Athens, Greece; \\ lrallidis@gmail.com \\ * Correspondence: dbpanag@hua.gr; Tel.: +30-210-9549332
}

Academic Editors: Helieh S. Oz and Veeranoot Nissapatorn

Received: 11 April 2019; Accepted: 14 May 2019; Published: 15 May 2019

check for updates

\begin{abstract}
Tea is one of the most-widely consumed beverages in the world with a number of different beneficial health effects, mainly ascribed to the polyphenolic content of the tea catechins. The aim of this study was to examine the consumption of green, black, or no tea, in relation to the previously validated successful ageing index (SAI; higher values "healthier" ageing) in a combined analysis of adults aged $>50$ years old from the ATTICA $(n=1128$ adults from Athens, Greece metropolitan area) and the MEDiterranean Islands Study (MEDIS) $(n=2221$ adults from various Greek island and Mani) studies. After adjusting for age, sex, smoking, and coffee consumption, green tea was positively associated with SAI ( $\mathrm{b} \pm$ SE: $0.225 \pm 0.055, p<0.001)$, while black tea was negatively associated with SAI (unstandardized b coefficient \pm Standard error: $-0.807 \pm 0.054, p<0.001$ ). Green tea (vs black tea) consumption, had higher odds of a SAI of over 3.58 out of 10 (OR: 1.77, 95\% CI: 1.38-2.28). Green tea consumption was also associated with higher levels of physical activity $(p<0.001)$ and reduced likelihood of hypertension $(p=0.006)$ compared with black tea. Two possible mechanisms are that green tea possesses high levels of catechins such as (-)-epigallocatechin 3-gallate and L-theanine compared with black tea. Therefore, the present analysis supports both the role of green tea constituents in successful ageing, as well as its role as an important component of an overall healthy diet in adults aged 50 years and over from these two epidemiological studies.
\end{abstract}

Keywords: green tea; EGCG; tea consumption; successful ageing index; cardiovascular disease 


\section{Introduction}

Tea is one of the most widely consumed beverages worldwide, and global consumption ranks second only to water, well ahead of coffee, beer, wine and carbonated soft drinks [1,2]. Traditionally, the Chinese and Japanese people have believed that drinking tea promotes optimal health and longevity [3,4]. Therefore, it is not surprising that the health-promoting effects of tea have been intensively investigated over recent decades [3,5,6]. Prior to consumption, tea is generally processed and relatively recent research has classified tea originating from the plant Camellia sinensis into seven types based on the type of processing as; 'green' (unfermented), 'yellow', 'white', 'oolong' (partially fermented), 'black' (completely fermented), 'aged pu-erh' (drastically fermented and aged) and 'ripened pu-erh' tea $[7,8]$. The global consumption of tea type varies, with black tea the most popular and favored in Western countries [9], green tea favored in Oriental countries, and oolong and pu-erh tea predominately consumed in China $[3,10,11]$.

The manufacture of black tea involves the oxidative polymerization of the monomeric flavan-3-ols by the enzyme polyphenol oxidase, leading to the formation of bisflavanols, theaflavins, thearubigins and other oligomers. In contrast, during the production of green tea, freshly harvested leaves are quickly steamed or hot air-dried to inhibit the oxidizing enzyme, polyphenol oxidase, which prevents fermentation of the tea, yielding a dry and stable product. The blanching of leaves caused by this exposure of tea to hot steam or air is also responsible for the production of its pronounced green color $[1,6]$. The composition of green tea varies with the tea plant variety, harvest season, the position of the plucked leaves, horticultural practices, climate and geographical position [11]. However, all green teas are rich in polyphenols, including flavadiols, flavonoids and phenolic acids. The beneficial health effects of green tea are primarily ascribed to the biologically active polyphenolic components, especially the monomeric flavan-3-ols and the catechins [3,10-12].

Catechins are the most prominent and biologically active compounds in green tea, almost all of which are extracted during the tea brewing process. These compounds also provide a slightly astringent and bitter taste to green tea infusions with one cup $(2.5 \mathrm{~g} / 200 \mathrm{~mL})$ containing approximately 150-200 mg of catechins in total [6]. The content of catechins in green tea can reach up to $30 \%$ of the tea leaves' dry weight [13] with (-)-epigallocatechin 3-gallate (EGCG) being the most predominant, accounting for up to $60 \%$ of the total catechin content [14]. Nevertheless, the (-)-epigallocatechin (EGC) was also reported to be one of the major catechins found in several green tea samples [15,16], making EGCG and EGC, undoubtedly the major catechins present in green tea infusions. However, oolong, black and pu-erh teas are allowed to be fermented and oxidized, lowering their catechin content due to their conversion to theaflavins and thearubigins [3].

Green tea polyphenols are predominately comprised of four major catechins; in decreasing order of the amount present in tea, are: EGCG; (-)-epigallocatechin (EGC); (-)-epicatechin 3-gallate (ECG) and (-)-epicatechin (EC) $[6,11]$. The structure of these four catechins (Figure 1) is characterized by multiple hydroxyl groups on two benzene rings. The gallocatechins (EGC and EGCG) have an extra hydroxyl group in the $5^{\prime}$ position of the B ring while the catechin gallates (ECG and EGCG) have an extra benzene ring with three hydroxyl groups [17]. 


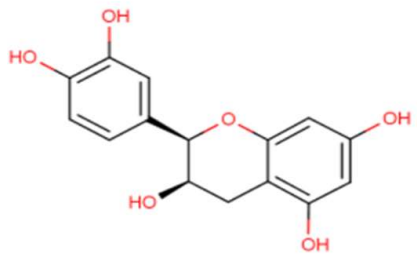

(a)

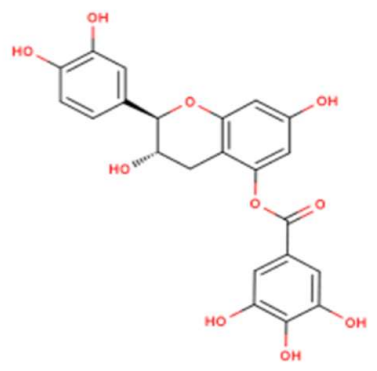

(c)<smiles>Oc1cc(O)c2c(c1)OC(c1cc(O)c(O)c(O)c1)C(O)C2</smiles>

(b)

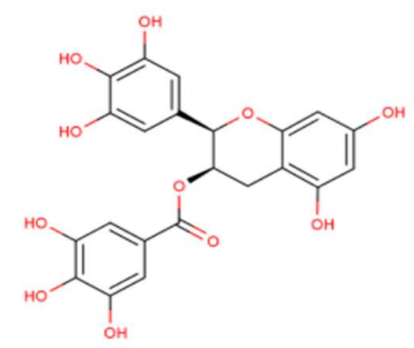

(d)

Figure 1. Chemical structures of the four main green tea catechins (a) Epicatechin; (b) Epigallocatechin; (c) Epicatechin-3-gallate; (d) Epigallocatechin-3-gallate. Adapted from [6,18].

There is increasing evidence from epidemiological, clinical and experimental studies which suggest that the tea polyphenols (EGCG in particular) have various biological activities such as anti-fungal [19], anti-inflammatory $[20,21]$ and antioxidative [22] properties. Habitual green tea consumption is associated with protection against several types of cancer [23] and lower all-cause mortality [24], while total consumption of tea has shown beneficial changes in psycho-cardiological outcomes, such as reduced risk of cardiovascular disease [25] and type 2 diabetes [26], lower blood pressure, reduced risk of developing hypertension in older adulthood [27], and decreased risk of cognitive impairment [28]. Black tea consumption is also associated with decreased risk of the development of cardiovascular disease in a 'normal' population [29]. Interestingly, two relatively recent meta-analyses of randomized controlled trials of black tea consumption on concentrations of serum cholesterols reported conflicting results with one showing no beneficial effects [30], and the other finding lower low density lipoprotein cholesterol (LDL-C) [31]. Nevertheless, higher tea consumption is considered part of a healthy lifestyle that may benefit health through various ways [25,29].

Aging is a continuous process associated with a higher likelihood of chronic disease and risk of increased morbidity and mortality [32]. The term successful aging incorporates many aspects of healthy living including quality of life, well-being, physical health, and the maintenance of cognitive function [33]. The role of dietary patterns on successful ageing is becoming better understood, with a current focus on the Mediterranean diet and its predominant constituents which include fruits and vegetables [34], and olive oil [35]. However, the potential health amplifying effects of tea consumption in the context of a Mediterranean style dietary pattern are unclear. Therefore, this study aimed to examine the association of tea consumption (green, black and no tea) on successful aging in people aged 50 years and over, living in Greece.

\section{Results}

In Table 1 some of the socio-demographic data, lifestyle and clinical characteristics of the ATTICA and MEDIS participants, categorized by type of tea consumption, are presented and used for the analyses. From the total of 1195 participants consuming tea, participants with green tea consumption were more frequently male $(p=0.026)$ and physically active $(p<0.001)$, while they were less likely to have hypertension $(p=0.006)$ and more likely to have a higher level of the successful aging index (SAI) 
$(p<0.001)$, as compared to those consuming black tea. No statistically significant differences were observed as regards cardiometabolic risk factors, adherence to the Mediterranean diet and smoking habits (all $\left.p^{\prime} \mathrm{s}>0.05\right)$.

Table 1. Sociodemographic, lifestyle, and clinical characteristics of the ATTICA and MEDIS study participants based on the type of tea (green/black) they consume.

\begin{tabular}{|c|c|c|c|c|}
\hline & $\begin{array}{l}\text { MEDIS and } \\
\text { ATTICA Study } \\
\text { Participants * } \\
\quad(n=3349)\end{array}$ & $\begin{array}{c}\text { Green Tea } \\
\text { Consumers } \\
(n=595)\end{array}$ & $\begin{array}{l}\text { Black Tea } \\
\text { Consumers } \\
(n=600)\end{array}$ & $p$ \\
\hline Age (years) & $69 \pm 10$ & $74 \pm 8.3$ & $74 \pm 7.0$ & 0.300 \\
\hline Male n (\%) & $1751(52)$ & $318(53)$ & $281(47)$ & 0.026 \\
\hline Female n (\%) & $1598(48)$ & $277(47)$ & $319(53)$ & 0.026 \\
\hline Physically active $\mathrm{n}(\%)$ & $1372(41)$ & $278(47)$ & $219(37)$ & $<0.001$ \\
\hline BMI $\left(\mathrm{kg} / \mathrm{m}^{2}\right)$ & $28 \pm 4.4$ & $29 \pm 4.7$ & $29 \pm 4.8$ & 0.129 \\
\hline Hypertension n (\%yes) & $1881(86)$ & $362(89)$ & $423(94)$ & 0.006 \\
\hline Diabetes n (\%yes) & $696(21)$ & $146(25)$ & $139(23)$ & 0.578 \\
\hline Hypercholesterolemia n (\%yes) & $1747(53)$ & $327(55)$ & 317 (53) & 0.458 \\
\hline \multicolumn{5}{|l|}{ Frequency of tea consumption } \\
\hline $1-2$ cups & $238(20)$ & $124(21)$ & $114(18)$ & $<0.001$ \\
\hline $3-5$ cups & $124(10)$ & $68(11)$ & $56(8)$ & $<0.001$ \\
\hline $5+$ cups & $34(3)$ & $14(2)$ & $20(1)$ & 0.001 \\
\hline MedDietScore (0-55) & $29 \pm 7.1$ & $33 \pm 4.6$ & $33 \pm 4.9$ & 0.868 \\
\hline Cardiometabolic risk factors $(0-4)$ & $1.6 \pm 1.1$ & $1.7 \pm 1.0$ & $1.9 \pm 1.1$ & 0.092 \\
\hline SAI (0-10) & $3.1 \pm 1.2$ & $2.9 \pm 1.4$ & $2.3 \pm 1.3$ & $<0.001$ \\
\hline
\end{tabular}

* All MEDIS and ATTICA study participants consuming or not tea, or without information about tea consumption. Values are presented as percent $(\%)$ or mean \pm standard deviation. $p$ : probability values between green tea and black tea consumers derived from Student's $t$-test for continuous variables or the chi-square test for the categorical variables.

A significant correlation was observed between the type of tea consumed and SAI score (Spearman rho $=-0.232, p<0.001)$; thus, to further test the research hypothesis, multiple linear regression analysis was then applied (Table 2). After adjustments (Table 2) for age, sex, smoking habits and coffee intake, drinking, only black tea was negatively associated with SAI levels ( $b \pm$ SE: $-0.727 \pm 0.055, p<0.001$ ), whereas drinking green tea vs black tea was positively associated with SAI levels (b \pm SE: $0.503 \pm 0.078$, $p<0.001)$. Additionally, drinking exclusively green tea was positively associated with SAI levels (b \pm SE: $0.225 \pm 0.055, p<0.001$ ) compared to not drinking at all tea (control group), whereas drinking black tea vs no drinking at all tea was inversely associated with SAI levels $(p<0.001)$. Moreover, drinking green tea but no black or not drinking at all tea was not significantly associated with SAI levels $(p=0.722)$. Black tea consumption compared with no tea at all was negatively associated with SAI levels ( $\mathrm{b} \pm \mathrm{SE}:-0.807 \pm 0.054, p<0.001)$. Therefore, it is concluded that the consumption of green tea among individuals aged $>50$ years is associated with more favorable SAI levels (Table 2).

Table 2. Results from five linear regression models that evaluated the association between the type (Green or Black) of tea consumed and successful aging (dependent outcome), after adjusting for age, sex, smoking and coffee consumption, among ATTICA and MEDIS study participants.

\begin{tabular}{rcc}
\hline & $\mathbf{b} \pm \mathrm{SE}$ & $p$ \\
\hline Model 1 adj. for age, sex, smoking and coffee consumption + Green vs. Black tea & $0.503 \pm 0.078$ & $<0.001$ \\
Model 2 adj. for age, sex, smoking and coffee consumption + Green vs. Black tea or Control group & $0.020 \pm 0.057$ & 0.722 \\
Model 3 adj. for age, sex, smoking and coffee consumption + Green tea vs. Control group & $0.225 \pm 0.055$ & $<0.001$ \\
Model 4 adj. for age, sex, smoking and coffee consumption + Black vs. Green tea or Control group & $-0.727 \pm 0.055$ & $<0.001$ \\
Model 5 adj. for age, sex, smoking and coffee consumption + Black tea vs. Control group & $-0.807 \pm 0.054$ & $<0.001$ \\
\hline
\end{tabular}

Results for the association of tea consumption (yes/no) on SAI (i.e., the higher the better) are presented as unstandardized $\mathrm{b}$ coefficients \pm Standard Error and $p$-value. Control group: no tea drinking. 
In Table 3, results are presented from logistic regression models that evaluated the association between the type of tea and the SAI level. Regarding the overall sample, those drinking green tea had 1.77 times higher odds (95\%CI, 1.38-2.28) for having SAI over 3.58 compared to those drinking black tea, while those drinking green tea had 1.41 times higher odds (95\% CI, 1.15-1.73) for having SAI over the median compared to those drinking black tea or the control group. Moreover, regarding those drinking black tea, the odds of having high SAI levels were 38\% less than those drinking green or the control group. Similar logistic regression models based on gender showed that females and males drinking green tea had 1.75 and 1.79 times, respectively, higher odds (95\%CI, 1.22-2.52 and 1.26-2.53 respectively) for having SAI over 3.58 compared to those females and males drinking black tea. Accordingly, those females and males drinking green tea had 1.36 and 1.50 times, respectively, higher odds (95\% CI, 1.01-1.84 and 1.12-2.01 respectively) for having SAI over the median compared to those females and males drinking black tea or the control group. Finally, regarding those females and males drinking black tea, the odds of having high SAI levels were $38 \%$ and 33\% less than those females and males drinking green or the control group, respectively.

Table 3. Results from logistic regression models that evaluated the association between the type (Green or Black) of tea consumed with successful aging index (i.e., above or below the median value 3.58) (dependent variable) among ATTICA and MEDIS study participants, separately for males and females.

\begin{tabular}{|c|c|c|c|}
\hline & OR & $95 \% \mathrm{CI}$ & $p$ \\
\hline \multicolumn{4}{|l|}{ All Participants } \\
\hline Model 1 adj. for age, sex, smoking and coffee consumption + Green vs. Black tea & 1.77 & $1.38-2.28$ & $<0.001$ \\
\hline Model 3 adj. for age, sex, smoking and coffee consumption + Green tea vs. Control group & 1.20 & $1.05-1.51$ & 0.048 \\
\hline Model 4 adj. for age, sex, smoking and coffee consumption + Black vs. Green tea or Control group & 0.62 & $0.51-0.77$ & $<0.001$ \\
\hline Model 5 adj. for age, sex, smoking and coffee consumption + Black tea vs. Control group & 0.69 & $0.55-0.86$ & 0.001 \\
\hline Model 1 adj. for age, sex, smoking and coffee consumption + Green vs. Black tea & 1.75 & $1.22-2.52$ & 0.003 \\
\hline Model 2 adj. for age, sex, smoking and coffee consumption + Green vs. Black tea or Control group & 1.36 & $1.01-1.84$ & 0.049 \\
\hline Model 3 adj. for age, sex, smoking and coffee consumption + Green tea vs. Control group & 1.12 & $0.80-1.56$ & 0.527 \\
\hline Model 4 adj. for age, sex, smoking and coffee consumption + Black vs. Green tea or Control group & 0.62 & $0.46-0.83$ & 0.002 \\
\hline Model 5 adj. for age, sex, smoking and coffee consumption + Black tea vs. Control group & 0.63 & $0.45-0.89$ & 0.07 \\
\hline \multicolumn{4}{|l|}{ Males } \\
\hline Model 5 adj. for age, sex, smoking and coffee consumption + Black tea vs. Control group & 0.76 & $0.56-1.04$ & 0.087 \\
\hline
\end{tabular}

Results of tea consumption (yes/no) on the likelihood of having higher SAI (i.e., better aging) are presented as Odds Ratios (OR), their corresponding $95 \% \mathrm{CI}$ and $p$-value.

\section{Discussion}

The present pooled analysis of two large-scale population-based studies conducted in Greece assessed the association between green and black tea consumption on successful aging in older people. The main findings demonstrated that the consumption of green tea was linked to a higher likelihood of successful aging based on the SAI compared with consumption of black tea following adjustment for potential confounding factors including age, sex, smoking, and coffee consumption. Interestingly, black tea consumption was negatively associated with successful aging compared with both green tea consumption and no tea consumption. Therefore, green tea, but not black tea, may be associated with enhanced healthy aging in older people in this population living in the Mediterranean region.

While both green tea and black tea are widely considered as healthy beverages, only green tea was associated with a higher SAI while in contrast, black tea was associated with a lower SAI. No differences in average daily consumption of the number of participants consuming green and black tea were observed. Although both green and black tea are high in antioxidants such as polyphenols and flavonoids, green tea possesses high amounts of catechins such as EGCG. In human trials, EGCG has been shown to promote fat oxidation and increase postprandial thermogenesis [36], while green 
tea consumption promotes lower total and LDL-cholesterol [37], and possesses anti-inflammatory properties [38]. When considering all these potential mechanisms of action and beneficial health effects of green tea consumption, it is intriguing that consumption of green tea is associated with the higher SAI. A relatively recent systematic review of the effects of green tea catechins on cardiovascular disease risk factors in human clinical trials suggests that BMI, hypertension, and plasma lipids may be improved, but several methodological issues limit the generalizability of the current evidence [6].

Although observed results relating to the differences between black and green tea are inconsistent, these observational associations could be potentially supported by substantial evidence from experimental studies investigating the antioxidant and vasodilation effects of the various tea compounds such as the L-theanine (L-THE) [1,2,39]. The L-THE is non-proteinous, and the most predominant amino acid found in green tea, accounting for over $50 \%$ of the total amino acid content. Concurrent with increasing commercial availability of the relatively pure L-THE [39], dose-dependent clinical trials have identified its hypotensive properties and negation of caffeine-induced effects related to increases in blood pressure [2]. Tea processing such as withering and fermentation are commonly applied to different tea varieties and only marginally influences the caffeine content [40], suggesting the importance of L-THE in the prevention of caffeine-related side effects. Green tea also contains gamma-aminobutyric acid (GABA) which in animal models has been shown to decrease blood pressure [41] and improve antioxidative defenses and mood status [42] through the modulations of neurotransmitters responsible for antidepressant-like effects [43]. Besides the content of L-THE and GABA, as well as many other unidentified potentially biologically active compounds present in tea [44], there are several other proposed mechanisms of action related to green tea consumption such as the suppression of the NADPH oxidase activity and reduction of reactive oxygen species in the vascular system [45]. Green tea polyphenols also possess anti-inflammatory properties in vitro through blocking of NFKB activation [46], and in animal models has been shown to decrease production of IL-6, TNF $\alpha$, serum amyloid A [47] and S100ß [48].

The positive results associated with green tea are potentially explained by the differences in the compositions of the two different tea varieties. It is well established that green tea contains higher levels of catechins in comparison to black tea, primarily due to the processing of tea leaves post-harvest [11,17]. Therefore, these higher levels of green tea catechins could be the driving factor for the lower levels of hypertension in this population sample, and similar findings have been reported in different population settings. For example, in Taiwanese men and women [49], habitual intake of green or oolong tea over $120 \mathrm{~mL} /$ day was associated with a reduced risk of developing hypertension. A recent systematic literature review with meta-analysis analyzed studies where green tea extracts high in catechins reduce systolic blood pressure, and total and LDL cholesterol levels [45]. Consumption of both tea varieties (black and green) can be beneficial in lowering blood pressure in individuals with prehypertensive and hypertensive ranges [50,51]. On the other hand, acute consumption of caffeine found in green and black tea may increase blood pressure, in participants who avoided caffeine for at least $12 \mathrm{~h}[52,53]$. However, the relevance of these acute trials to regular tea consumption over the long term are still uncertain. Interestingly, short-term controlled clinical trials of regular tea consumption up to 8 weeks in normotensive individuals have shown that regular tea consumption does not have an overall effect on systolic or diastolic blood pressures [54,55]. Nevertheless, it is plausible that longer-term habitual consumption of tea can positively affect vasodilatory function and consequently results in altered vascular tone and blood pressure [56].

The population sample in the present study that consumed higher levels of green tea presented with fewer cases of hypertension but were also more physically active and also more likely to be male. Taking into consideration that SAI is constructed using ten attributes including the physical activity, BMI, depression, cardiovascular disease risk factors, as well as adherence to Mediterranean diet [57], it is intriguing that green tea consumption has shown higher odds for having SAI if compared to those of drinking black tea. Both groups consuming black and green tea had greater adherence to a Mediterranean diet pattern compared with the overall sample. Therefore, the divergent results 
for each type of tea were not confounded by the overall dietary pattern. In addition, prevalence of hypercholesterolemia and other cardiometabolic risk factors were not different between the types of tea. As such, it is plausible that green tea could be considered as a proxy for an overall healthier lifestyle in this population, and the results may be confounded by healthy user bias in green tea consumers, leading to a higher rating of the SAI. In particular, physical activity represents a potential confounding variable as it indicates green tea drinkers may be more health conscious, however, the green tea group also contained more smokers compared with black tea group, although the difference was not statistically significant.

Importantly, when proposing potential beneficial health effects of tea (and tea beverages) the quantity and the stability of the tea catechins (and other compounds) during the consumption and preparation of the beverages must be considered. From this perspective, temperature and exposure time (brewing time) pose an essential role in the stability of the tea components [58] as much as the quality of the water used in the preparation of a tea [59] and the repeated use of water during the tea preparation [60]. The latter is more prevalent (and somewhat exclusive) in Asian societies, making it very hard to count accurately the number of cups as a delivery method of tea flavanols (catechins) as the measure of exposure.

To our knowledge, this is one of the first studies to investigate the consumption of green and black tea, and tea overall, in the Mediterranean region in relation to the successful ageing of the older adults. Two large population-based samples were analyzed in combination, reducing the potential for bias and increasing the external validity of the findings. A strength of the study is also the adjustment for confounding based on coffee consumption. The effects of coffee on overall health are relatively controversial but have been associated with lower all-cause mortality [61]. Nevertheless, this study has several limitations. Dietary habits and participants data were only measured through one food frequency questionnaires (FFQ) with potential for bias towards an inaccurate recall of food intake or tendency to over-report 'healthy' foods such as green tea. The FFQ could not evaluate the main catechins, polymerized polyphenols, and amino acid compositions in the observed teas. Therefore, the direct effects of these constituents cannot be readily established. However, adherence to a Mediterranean style dietary pattern was similar in both green and black tea drinkers, strengthening the findings of benefits due to green tea consumption. Another limitation of the analysis is both consumers of green and black tea scored lower on the SAI than the overall population sample which may be related to the higher age of both tea drinking groups. Higher percentages of hypertension, diabetes, and hypercholesterolemia were also observed in the tea drinking groups, which may also be contributing to the lower SAI score compared with the overall sample.

In conclusion, the consumption of green tea was associated with a higher likelihood of successful aging in a population sample in Greece. Interestingly, consumption of black tea was negatively associated with successful ageing and potential reasons for this observation definitely raise several questions related to the chemical composition of the two different tea varieties. The positive effects of green tea may be associated with their higher levels of catechins and other predominant constituents such as L-THE, GABA and other biologically active compounds. However, green tea consumption was also associated with higher levels of physical activity and lower prevalence of hypertension, suggesting green tea is consumed by individuals living an overall healthier lifestyle in the region.

\section{Materials and Methods}

The study sample consisted of participants of the ATTICA and MEDIS studies, aged $>50$ years old, residing in urban and insular Greek areas. As previously detailed [62], the ATTICA study was a population-based observational study implemented in the greater metropolitan Athens area, during 2001-2002. At baseline, all participants were free of CVD and cancer, as assessed through a detailed clinical evaluation by the study's physicians. From the original sample $(n=2583)$ over 18 years old, of the ATTICA sample, a sub-group of $n=1128$ individuals aged $>50$ years old were analyzed for the purposes of the present work. In addition, as previously detailed [57], based on the MEDIS study, 
approximately 3000 older people from Mani (Greek peninsula region) and 26 Mediterranean islands of 5 countries were enrolled during 2005-2017 (MEDIS study). Individuals who resided in assisted-living centers, had a clinical history of cardiovascular disease (CVD) or cancer, or had left the island for a considerable period of time during their life (i.e., $>5$ years) were excluded. Of the 3138 MEDIS study participants aged $>50$ years living in the insular Mediterranean region (Cyprus, Spain, Italy, Turkey and Greece), a sub-group of $n=2221$ individuals from Greek islands were analyzed in this work. For both aforementioned studies, a group of trained health scientists (including cardiologists, general practitioners, physicians, dietitians, public health nutritionists, and nurses) collected all information using standard, validated questionnaires and clinical procedures.

\subsection{Bioethics}

The ATTICA study was approved by the Bioethics Committee of Athens Medical School and was carried out in accordance with the Declaration of Helsinki (1989) of the World Medical Association. The MEDIS study was approved by the Institutional Ethics Board of Harokopio University (16/19-12-2006) and followed the ethical recommendations of the World Medical Association (52nd WMA General Assembly, Edinburgh, Scotland, October 2000). In both studies, participants were informed of the study aims and procedures, and provided written informed consent for study participation prior to enrolment.

\subsection{Measurements}

\subsubsection{Sociodemographic Data}

The sociodemographic characteristics assessed within the context of the present investigation were age (years), gender (male/female), and smoking status. Current smokers were defined as those who smoked at least one cigarette or any type of tobacco per day at the time of the interview. Former smokers were defined as those who previously smoked but had quit within the previous year. Current and former smokers were combined as ever smokers. The remaining participants were defined as non-smokers.

\subsubsection{Physical Activity Levels}

Physical activity was evaluated in Metabolic equivalent (MET) minutes per week, using the shortened, translated and validated in Greek version of the self-reported International Physical Activity Questionnaire (IPAQ) [62]. Those who reported at least 3 MET-minutes per week were classified as physically active. All others were defined as physically inactive.

\subsubsection{Anthropometric and Clinical Characteristics}

Weight and height were measured using standard procedures to attain volunteer's Body Mass Index (BMI) $\left(\mathrm{kg} / \mathrm{m}^{2}\right)$. Overweight was defined as BMI between 25.0 and $29.9 \mathrm{~kg} / \mathrm{m}^{2}$, while obesity was defined as BMI $>29.9 \mathrm{~kg} / \mathrm{m}^{2}$. Type 2 diabetes mellitus (T2DM) was determined by measuring fasting plasma glucose and in accordance with the American Diabetes Association diagnostic criteria (fasting blood glucose $>126 \mathrm{mg} / \mathrm{dL}$ or use of antidiabetic medication). Participants who had blood pressure levels $>140 / 90 \mathrm{~mm} \mathrm{Hg}$ or who were administered antihypertensive medications were classified as hypertensive. Fasting blood lipids levels were also recorded. Hypercholesterolemia was defined as total serum cholesterol levels $>200 \mathrm{mg} / \mathrm{dL}$ or the use of lipid-lowering agents according to the National Cholesterol Education Program Adult Treatment Panel III guidelines [63]. The coefficient of variation for the blood measurements was less than 5\%. A cumulative indicator of Cardiometabolic Risk, indicating the overall burden of known cardiometabolic risk factors (i.e., obesity and history of hypertension, T2DM, and hypercholesterolemia) was constructed (score range 0-4), wherein participants having none of the aforementioned risk factors were assigned a score of 0 , having one factor a score of 1 , etc. 


\subsubsection{Dietary Habits Assessment}

Among ATTICA study participants, the evaluation of dietary habits was based on a semi-quantitative food-frequency questionnaire (FFQ), originally developed for the European Prospective Investigation into Cancer and Nutrition (EPIC) study [64]. The Greek version of the EPIC questionnaire was provided by the Unit of Nutrition of Athens Medical School, after being translated according to standard literature guidelines [65]. Participants were requested to report the average intake (per week or per day) of several food items that they have been consuming (during the last 12 months). Similar to the ATTICA study, dietary habits in the MEDIS study were assessed through a semi-quantitative, validated, and reproducible FFQ [66]. In both studies, consumption of various food groups and beverages (i.e., meat and meat products, poultry, fish, milk and other dairy products, fruits, vegetables, greens and salads, legumes, cereals, pasta, soft drinks, coffee and tea) was measured, in times of weekly consumption (never, rare, 2-3 times/month, 1-2 times/week, 3-5 times per week and daily). Particularly for tea consumption all participants were asked about the type of tea (green/black) and the frequency they consume a cup (of $150 \mathrm{~mL}$ ) within a week (i.e., never, $<1$ cup/week, 1-2 cups/day, $3-5$ cups/day, $>5$ cups/day. Moreover, overall assessment of dietary habits was evaluated through MedDietScore (range 0-55) that assesses adherence to the Mediterranean dietary pattern [67].

\subsubsection{Successful Aging Index}

A Successful Aging Index (SAI), ranging from 0 to 10, which has been previously developed and validated [57], using 10 attributes that reflect and have been found associated with the aging process, was applied for assessing successful ageing. The index encompasses health-related social-, lifestyle- and clinical factors, including education, financial status, physical activity, BMI, depression, participation in social activities with friends and family, number of yearly excursions, total number of clinical CVD risk factors (i.e., history of hypertension, diabetes, hypercholesterolemia, obesity) and level of adherence to the Mediterranean diet. Specifically: Education (years of school): 0-2 years $=0,3-6$ years $=0.33,7-12$ years $=0.66,>12$ years $=1$, Financial status (self-reported): Low $=0$, moderate $=0.33$, good $=0.66$, very good $=1$, Social activities with friends (per week): None $=0,1$ time $=0.25,2$ times $=0.5$, $3-5$ times $=0.75,>5$ times $=1$, Social activities with family (per week): None $=0,1$ time $=0.25$, 2 times $=0.5,3-5$ times $=0.75,>5$ times $=1$, Going to excursions: None $=0,1$ time $=0.25,2$ times $=0.5$, $3-5$ times $=0.75,>5$ times $=1$, Physical activity (per week): None $=0,1-2$ times $=0.33,3-5$ times $=0.66$, $>5$ times $=1$, BMI classes: Normal weight: 1 , overweight $=0.5$, underweight or obese $=0$, Geriatric Depression Scale (GDS) (0-15): 0-4 points (no depression) $=1,5-10$ points (mild depression) $=0.5$, $>10$ points (severe depression) $=0$, CVD risk score (i.e., obesity, history of hypertension, diabetes, hypercholesterolemia): None $=1,1$ factor $=0.75,2$ factors $=0.5,3$ factors $=0.25,4$ factors $=0$, and Level of adherence to the Mediterranean diet (MedDietScore 0-55): 0-34 points $=0,35-38$ points $=0.5$, $>38$ points $=1$.

\subsection{Statistical Analysis}

Continuous variables are presented as mean \pm standard deviation $(\mathrm{SD}$, and categorical variables as frequencies. Associations between continuous variables and group of participants were evaluated with Student's $t$-test; Spearman rho correlation coefficient was used to evaluate relationships between cups of tea drinking and SAI score. Multiple linear regression models were used to evaluate the associations between tea consumption (independent variables), adjusted for various participants' characteristics (i.e., age, gender, coffee consumption and smoking habits) and the SAI (dependent outcome). The Results are presented as unstandardized beta coefficients \pm standard error $(b \pm S E)$ and $p$-value. Linearity of models' fitting was tested through the scatter plots of standardized residuals against fitted values; normality of regression residuals was evaluated through P-P plots; dependency was tested using the Durbin-Watson test and homoscedacity using the Variance Inflation Index (value $<4$ suggests lack of heteroscedacity). Moreover, multiple binary logistic regression models were used 
to evaluate the association between the type of tea (Green vs Black tea, Green vs Black or no tea at all [control group], and Black vs Green or control group) (independent variables) with the level of successful aging index (i.e., above or below the median value 3.58) (dependent variable). The results are presented as Odds Ratios (OR) of the likelihood of having SAI above the median value and their 95\% confidence intervals. Deviance residuals were used to evaluate models' goodness-of-fit. Tea consumption was assessed as "drinking" or "not-drinking" (i.e., yes/no) in all multi-adjusted analyses because of lack adequate data in each consumption category. STATA (College Station, TX, USA \& M. Psarros et Assoc., Sparta, Greece) software version 13 was used for all calculations.

Author Contributions: Conceptualization, N.N. and D.P.; Formal analysis, A.F.; Methodology, A.F., E.P. and D.P.; Project administration, D.P.; Supervision, N.N. and D.P.; Visualization, N.N., A.F. and N.M.D.; Writing-original draft, N.M.D.; Writing—review \& editing, N.N., A.F., N.M.D., S.T., C.C., L.S.S., L.R., A.-L.M., C.P. and D.P.

Funding: The ATTICA study is supported by research grants from the Hellenic Cardiology Society (HCS2002) and the Hellenic Atherosclerosis Society (HAS2003). The MEDIS study was funded by Research grants from the Hellenic Heart Foundation, the Graduate Program of the Department of Nutrition \& Dietetics, Harokopio University and the Rutgers University, NJ, USA (GA \#5884). Stefanos Tyrovolas was supported by the Foundation for Education and European Culture (IPEP), the Sara Borrell postdoctoral program (reference no. CD15/00019 from the Instituto de Salud Carlos III (ISCIII - Spain) and the Fondos Europeo de Desarrollo Regional (FEDER). Demosthenes Panagiotakos, Stefano Tyrovolas and Alexandra Foscolou have been funded for ATHLOS project to study trajectories of healthy aging (European Union's Horizon 2020 research and innovation program, grant agreement No 635316).

Acknowledgments: Authors are particularly grateful to the men and women from all areas that participated in the ATTICA and MEDIS studies, as well as to both studies investigators.

Conflicts of Interest: The authors declare no conflict of interest.

\section{References}

1. Naumovski, N.; Blades, B.L.; Roach, P.D. Food Inhibits the Oral Bioavailability of the Major Green Tea Antioxidant Epigallocatechin Gallate in Humans. Antioxidants (Basel) 2015, 4, 373-393. [CrossRef]

2. Williams, J.; Sergi, D.; McKune, A.J.; Georgousopoulou, E.N.; Mellor, D.D.; Naumovski, N. The beneficial health effects of green tea amino acid l-theanine in animal models: Promises and prospects for human trials. Phytother. Res. 2019, 33, 571-583. [CrossRef] [PubMed]

3. Lin, J.K.; Lin-Shiau, S.Y. Mechanisms of hypolipidemic and anti-obesity effects of tea and tea polyphenols. Mol. Nutr. Food Res. 2006, 50, 211-217. [CrossRef]

4. Yang, C.S.; Landau, J.M. Effects of tea consumption on nutrition and health. J. Nutr. 2000, 130, $2409-2412$. [CrossRef]

5. Huang, H.; Han, G.Y.; Jing, L.P.; Chen, Z.Y.; Chen, Y.M.; Xiao, S.M. Tea Consumption Is Associated with Increased Bone Strength in Middle-Aged and Elderly Chinese Women. J. Nutr. Health Aging 2018, 22, $216-221$. [CrossRef]

6. Lau, S.O.; Georgousopoulou, E.N.; Kellett, J.; Thomas, J.; McKune, A.; Mellor, D.D.; Roach, P.D.; Naumovski, N. The Effect of Dietary Supplementation of Green Tea Catechins on Cardiovascular Disease Risk Markers in Humans: A Systematic Review of Clinical Trials. Beverages 2016, 2, 16. [CrossRef]

7. Yi, T.; Zhu, L.; Peng, W.-L.; He, X.-C.; Chen, H.-L.; Li, J.; Yu, T.; Liang, Z.-T.; Zhao, Z.-Z.; Chen, H.-B. Comparison of ten major constituents in seven types of processed tea using HPLC-DAD-MS followed by principal component and hierarchical cluster analysis. LWT Food Sci. Technol. 2015, 62, 194-201. [CrossRef]

8. Koch, W.; Kukula-Koch, W.; Komsta, L. Black Tea Samples Origin Discrimination Using Analytical Investigations of Secondary Metabolites, Antiradical Scavenging Activity and Chemometric Approach. Molecules 2018, 23, 513. [CrossRef] [PubMed]

9. Ng, T.P.; Aung, K.C.; Feng, L.; Feng, L.; Nyunt, M.S.; Yap, K.B. Tea consumption and physical function in older adults: A cross-sectional study. J. Nutr. Health Aging 2014, 18, 161-166. [CrossRef]

10. Crespy, V.; Williamson, G. A review of the health effects of green tea catechins in in vivo animal models. J. Nutr. 2004, 134, 3431S-3440S. [CrossRef]

11. Lin, Y.S.; Tsai, Y.J.; Tsay, J.S.; Lin, J.K. Factors affecting the levels of tea polyphenols and caffeine in tea leaves. J. Agric. Food Chem. 2003, 51, 1864-1873. [CrossRef] 
12. Tokunaga, S.; White, I.R.; Frost, C.; Tanaka, K.; Kono, S.; Tokudome, S.; Akamatsu, T.; Moriyama, T.; Zakouji, H. Green tea consumption and serum lipids and lipoproteins in a population of healthy workers in Japan. Ann. Epidemiol. 2002, 12, 157-165. [CrossRef]

13. Kuo, K.L.; Weng, M.S.; Chiang, C.T.; Tsai, Y.J.; Lin-Shiau, S.Y.; Lin, J.K. Comparative studies on the hypolipidemic and growth suppressive effects of oolong, black, pu-erh, and green tea leaves in rats. J. Agric. Food Chem. 2005, 53, 480-489. [CrossRef]

14. Nagle, D.G.; Ferreira, D.; Zhou, Y.D. Epigallocatechin-3-gallate (EGCG): Chemical and biomedical perspectives. Phytochemistry 2006, 67, 1849-1855. [CrossRef]

15. Lin, S.-D.; Liu, E.-H.; Mau, J.-L. Effect of different brewing methods on antioxidant properties of steaming green tea. LWT Food Sci. Technol. 2008, 41, 1616-1623. [CrossRef]

16. Koch, W.; Kukula-Koch, W.; Komsta, L.; Marzec, Z.; Szwerc, W.; Glowniak, K. Green Tea Quality Evaluation Based on Its Catechins and Metals Composition in Combination with Chemometric Analysis. Molecules 2018, 23, 1689. [CrossRef]

17. Balentine, D.A.; Wiseman, S.A.; Bouwens, L.C. The chemistry of tea flavonoids. Crit. Rev. Food Sci. Nutr. 1997, 37, 693-704. [CrossRef]

18. Kim, S.; Thiessen, P.A.; Bolton, E.E.; Chen, J.; Fu, G.; Gindulyte, A.; Han, L.; He, J.; He, S.; Shoemaker, B.A.; et al. PubChem Substance and Compound databases. Nucleic Acids Res. 2016, 44, D1202-D1213. [CrossRef]

19. Hirasawa, M.; Takada, K. Multiple effects of green tea catechin on the antifungal activity of antimycotics against Candida albicans. J. Antimicrob. Chemother. 2004, 53, 225-229. [CrossRef]

20. Katiyar, S.K.; Mukhtar, H. Green tea polyphenol (-)-epigallocatechin-3-gallate treatment to mouse skin prevents UVB-induced infiltration of leukocytes, depletion of antigen-presenting cells, and oxidative stress. J. Leukoc. Biol. 2001, 69, 719-726.

21. Kawai, K.; Tsuno, N.H.; Kitayama, J.; Okaji, Y.; Yazawa, K.; Asakage, M.; Hori, N.; Watanabe, T.; Takahashi, K.; Nagawa, H. Epigallocatechin gallate attenuates adhesion and migration of CD8+ T cells by binding to CD11b. J. Allergy Clin. Immunol. 2004, 113, 1211-1217. [CrossRef]

22. Rietveld, A.; Wiseman, S. Antioxidant effects of tea: Evidence from human clinical trials. J. Nutr. 2003, 133, 3285S-3292S. [CrossRef]

23. Lambert, J.D.; Yang, C.S. Mechanisms of cancer prevention by tea constituents. J. Nutr. 2003, 133, 3262S-3267S. [CrossRef]

24. Kuriyama, S.; Shimazu, T.; Ohmori, K.; Kikuchi, N.; Nakaya, N.; Nishino, Y.; Tsubono, Y.; Tsuji, I. Green tea consumption and mortality due to cardiovascular disease, cancer, and all causes in Japan: The Ohsaki study. JAMA 2006, 296, 1255-1265. [CrossRef]

25. Giannakopoulou, S.P.; Panagiotakos, D. Can tea consumption reduce the risk of CVD? A discussion paper of a recently published cohort study. Hell. J. Atheroscler. 2018, 2018, 104-110.

26. Huxley, R.; Lee, C.M.; Barzi, F.; Timmermeister, L.; Czernichow, S.; Perkovic, V.; Grobbee, D.E.; Batty, D.; Woodward, M. Coffee, decaffeinated coffee, and tea consumption in relation to incident type 2 diabetes mellitus: A systematic review with meta-analysis. Arch. Intern. Med. 2009, 169, 2053-2063. [CrossRef]

27. Yin, J.Y.; Duan, S.Y.; Liu, F.C.; Yao, Q.K.; Tu, S.; Xu, Y.; Pan, C.W. Blood Pressure Is Associated with Tea Consumption: A Cross-sectional Study in a Rural, Elderly Population of Jiangsu China. J. Nutr. Health Aging 2017, 21, 1151-1159. [CrossRef]

28. Gu, Y.J.; He, C.H.; Li, S.; Zhang, S.Y.; Duan, S.Y.; Sun, H.P.; Shen, Y.P.; Xu, Y.; Yin, J.Y.; Pan, C.W. Tea consumption is associated with cognitive impairment in older Chinese adults. Aging Ment. Health 2018, 22, 1232-1238. [CrossRef]

29. Bahorun, T.; Luximon-Ramma, A.; Neergheen-Bhujun, V.S.; Gunness, T.K.; Googoolye, K.; Auger, C.; Crozier, A.; Aruoma, O.I. The effect of black tea on risk factors of cardiovascular disease in a normal population. Prev. Med. 2012, 54, S98-S102. [CrossRef]

30. Wang, D.; Chen, C.; Wang, Y.; Liu, J.; Lin, R. Effect of Black Tea Consumption on Blood Cholesterol: A Meta-Analysis of 15 Randomized Controlled Trials. PLoS ONE 2014, 9, e107711. [CrossRef]

31. Zhao, Y.; Asimi, S.; Wu, K.; Zheng, J.; Li, D. Black tea consumption and serum cholesterol concentration: Systematic review and meta-analysis of randomized controlled trials. Clin. Nutr. 2015, 34, 612-619. [CrossRef]

32. Franco, O.H.; Karnik, K.; Osborne, G.; Ordovas, J.M.; Catt, M.; van der Ouderaa, F. Changing course in ageing research: The healthy ageing phenotype. Maturitas 2009, 63, 13-19. [CrossRef] 
33. Milte, C.M.; McNaughton, S.A. Dietary patterns and successful ageing: A systematic review. Eur. J. Nutr. 2016, 55, 423-450. [CrossRef]

34. Foscolou, A.; Koloverou, E.; Matalas, A.-L.; Tyrovolas, S.; Chrysohoou, C.; Sidossis, L.; Rallidis, L.; Panagiotakos, D.B. Decomposition of Mediterranean Dietary Pattern on Successful Aging, Among Older Adults: A Combined Analysis of Two Epidemiological Studies. J. Aging Health 2018. [CrossRef]

35. Foscolou, A.; Critselis, E.; Tyrovolas, S.; Chrysohoou, C.; Sidossis, L.S.; Naumovski, N.; Matalas, A.L.; Rallidis, L.; Polychronopoulos, E.; Ayuso-Mateos, J.L.; et al. The Effect of Exclusive Olive Oil Consumption on Successful Aging: A Combined Analysis of the ATTICA and MEDIS Epidemiological Studies. Foods 2019, 8, 25. [CrossRef]

36. Boschmann, M.; Thielecke, F. The effects of epigallocatechin-3-gallate on thermogenesis and fat oxidation in obese men: A pilot study. J. Am. Coll. Nutr. 2007, 26, 389s-395s. [CrossRef]

37. Li, S.-H.; Zheng, X.-X.; Liu, X.-X.; Xu, Y.-L.; Huang, X.-H.; Hui, R. Green tea intake lowers fasting serum total and LDL cholesterol in adults: A meta-analysis of 14 randomized controlled trials. Am. J. Clin. Nutr. 2011, 94, 601-610. [CrossRef]

38. Ohishi, T.; Goto, S.; Monira, P.; Isemura, M.; Nakamura, Y. Anti-inflammatory Action of Green Tea. Antiinflamm. Antiallergy Agents Med. Chem. 2016, 15, 74-90. [CrossRef]

39. Williams, J.; Kellett, J.; Roach, P.D.; McKune, A.; Mellor, D.; Thomas, J.; Naumovski, N. L-Theanine as a Functional Food Additive: Its Role in Disease Prevention and Health Promotion. Beverages 2016, 2, 13. [CrossRef]

40. Caffin, N.; D’Arcy, B.; Yao, L.; Rintoul, G. Developing an Index of Quality for Australian Tea; Rural Industries Research and Development Corporation: Queensland, Australia, 2004.

41. Abe, Y.; Umemura, S.; Sugimoto, K.; Hirawa, N.; Kato, Y.; Yokoyama, N.; Yokoyama, T.; Iwai, J.; Ishii, M. Effect of green tea rich in gamma-aminobutyric acid on blood pressure of Dahl salt-sensitive rats. Am. J. Hypertens. 1995, 8, 74-79. [CrossRef]

42. Daglia, M.; Di Lorenzo, A.; Nabavi, S.F.; Sureda, A.; Khanjani, S.; Moghaddam, A.H.; Braidy, N.; Nabavi, S.M. Improvement of Antioxidant Defences and Mood Status by Oral GABA Tea Administration in a Mouse Model of Post-Stroke Depression. Nutrients 2017, 9, 446. [CrossRef]

43. Teng, J.; Zhou, W.; Zeng, Z.; Zhao, W.; Huang, Y.; Zhang, X. Quality components and antidepressant-like effects of GABA green tea. Food Funct. 2017, 8, 3311-3318. [CrossRef]

44. Bohn, S.K.; Ward, N.C.; Hodgson, J.M.; Croft, K.D. Effects of tea and coffee on cardiovascular disease risk. Food Funct. 2012, 3, 575-591. [CrossRef]

45. Onakpoya, I.; Spencer, E.; Heneghan, C.; Thompson, M. The effect of green tea on blood pressure and lipid profile: A systematic review and meta-analysis of randomized clinical trials. Nutr. Metab. Cardiovasc. Dis. 2014, 24, 823-836. [CrossRef]

46. Yang, F.; Oz, H.S.; Barve, S.; de Villiers, W.J.; McClain, C.J.; Varilek, G.W. The green tea polyphenol (-)-epigallocatechin-3-gallate blocks nuclear factor-kappa B activation by inhibiting I kappa B kinase activity in the intestinal epithelial cell line IEC-6. Mol. Pharmacol. 2001, 60, 528-533.

47. Oz, H.S.; Chen, T.; de Villiers, W.J.S. Green Tea Polyphenols and Sulfasalazine have Parallel Anti-Inflammatory Properties in Colitis Models. Front. Immunol. 2013, 4, 132. [CrossRef]

48. D’Cunha, N.M.; McKune, A.J.; Panagiotakos, D.B.; Georgousopoulou, E.N.; Thomas, J.; Mellor, D.D.; Naumovski, N. Evaluation of dietary and lifestyle changes as modifiers of S100beta levels in Alzheimer's disease. Nutr. Neurosci. 2019, 22, 1-18. [CrossRef]

49. Yang, Y.C.; Lu, F.H.; Wu, J.S.; Wu, C.H.; Chang, C.J. The protective effect of habitual tea consumption on hypertension. Arch. Intern. Med. 2004, 164, 1534-1540. [CrossRef]

50. Greyling, A.; Ras, R.T.; Zock, P.L.; Lorenz, M.; Hopman, M.T.; Thijssen, D.H.J.; Draijer, R. The effect of black tea on blood pressure: A systematic review with meta-analysis of randomized controlled trials. PLoS ONE 2014, 9, e103247. [CrossRef]

51. Yarmolinsky, J.; Gon, G.; Edwards, P. Effect of tea on blood pressure for secondary prevention of cardiovascular disease: A systematic review and meta-analysis of randomized controlled trials. Nutr. Rev. 2015, 73, $236-246$. [CrossRef]

52. Hodgson, J.M.; Burke, V.; Puddey, I.B. Acute effects of tea on fasting and postprandial vascular function and blood pressure in humans. J. Hypertens. 2005, 23, 47-54. [CrossRef] 
53. Pincomb, G.A.; Lovallo, W.R.; McKey, B.S.; Sung, B.H.; Passey, R.B.; Everson, S.A.; Wilson, M.F. Acute blood pressure elevations with caffeine in men with borderline systemic hypertension. Am. J. Cardiol. 1996, 77, 270-274. [CrossRef]

54. Hodgson, J.M. Effects of tea and tea flavonoids on endothelial function and blood pressure: A brief review. Clin. Exp. Pharmacol. Physiol. 2006, 33, 838-841. [CrossRef]

55. Hodgson, J.M. Tea flavonoids and cardiovascular disease. Asia Pac. J. Clin. Nutr. 2008, 17 (Suppl. 1), $288-290$.

56. Hodgson, J.M.; Croft, K.D. Tea flavonoids and cardiovascular health. Mol. Aspects Med. 2010, 31, 495-502. [CrossRef]

57. Tyrovolas, S.; Haro, J.M.; Mariolis, A.; Piscopo, S.; Valacchi, G.; Tsakountakis, N.; Zeimbekis, A.; Tyrovola, D.; Bountziouka, V.; Gotsis, E.; et al. Successful aging, dietary habits and health status of elderly individuals: A k-dimensional approach within the multi-national MEDIS study. Exp. Gerontol. 2014, 60, 57-63. [CrossRef]

58. Sang, S.; Lambert, J.D.; Ho, C.T.; Yang, C.S. The chemistry and biotransformation of tea constituents. Pharmacol. Res. 2011, 64, 87-99. [CrossRef]

59. Franks, M.; Lawrence, P.; Abbaspourrad, A.; Dando, R. The Influence of Water Composition on Flavor and Nutrient Extraction in Green and Black Tea. Nutrients 2019, 11, 80. [CrossRef]

60. Chei, C.L.; Loh, J.K.; Soh, A.; Yuan, J.M.; Koh, W.P. Coffee, tea, caffeine, and risk of hypertension: The Singapore Chinese Health Study. Eur. J. Nutr. 2018, 57, 1333-1342. [CrossRef]

61. Gunter, M.J.; Murphy, N.; Cross, A.J.; Dossus, L.; Dartois, L.; Fagherazzi, G.; Kaaks, R.; Kuhn, T.; Boeing, H.; Aleksandrova, K.; et al. Coffee Drinking and Mortality in 10 European Countries: A Multinational Cohort Study. Ann. Intern. Med. 2017, 167, 236-247. [CrossRef]

62. Panagiotakos, D.B.; Georgousopoulou, E.N.; Pitsavos, C.; Chrysohoou, C.; Metaxa, V.; Georgiopoulos, G.A.; Kalogeropoulou, K.; Tousoulis, D.; Stefanadis, C. Ten-year (2002-2012) cardiovascular disease incidence and all-cause mortality, in urban Greek population: The ATTICA Study. Int. J. Cardiol. 2015, 180, 178-184. [CrossRef] [PubMed]

63. Executive Summary of The Third Report of The National Cholesterol Education Program (NCEP) Expert Panel on Detection, Evaluation, And Treatment of High Blood Cholesterol In Adults (Adult Treatment Panel III). JAMA 2001, 285, 2486-2497. [CrossRef]

64. Katsouyanni, K.; Rimm, E.B.; Gnardellis, C.; Trichopoulos, D.; Polychronopoulos, E.; Trichopoulou, A. Reproducibility and relative validity of an extensive semi-quantitative food frequency questionnaire using dietary records and biochemical markers among Greek schoolteachers. Int. J. Epidemiol. 1997, 26 (Suppl. 1), S118-127. [CrossRef]

65. Maneesriwongul, W.; Dixon, J.K. Instrument translation process: A methods review. J. Adv. Nurs. 2004, 48, 175-186. [CrossRef] [PubMed]

66. Tyrovolas, S.; Pounis, G.; Bountziouka, V.; Polychronopoulos, E.; Panagiotakos, D.B. Repeatability and validation of a short, semi-quantitative food frequency questionnaire designed for older adults living in Mediterranean areas: The MEDIS-FFQ. J. Nutr. Elder. 2010, 29, 311-324. [CrossRef]

67. Panagiotakos, D.B.; Pitsavos, C.; Stefanadis, C. Dietary patterns: A Mediterranean diet score and its relation to clinical and biological markers of cardiovascular disease risk. Nutr. Metab. Cardiovasc. Dis. 2006, 16, 559-568. [CrossRef]

Sample Availability: Not Available. 